\title{
Computation of maximal magnetic field value generated by a power substation
}

\author{
N. Kovač ${ }^{1}$, D. Poljak ${ }^{1}$, S. Kraljević ${ }^{2}$, N. Božić ${ }^{2} \&$ N. Grulović ${ }^{1}$ \\ ${ }^{1}$ Split University, Croatia \\ ${ }^{2}$ Elektrodalmacija, Croatia
}

\begin{abstract}
A procedure for the computation of the maximal value of extremely low frequency (ELF) magnetic fields from a power substation is proposed. The present technique is based on the multiquadric approximation of the magnetic field. The approximation is obtained using discrete field values calculated by the Biot-Savart law, where each energised substation conductor has been divided into a corresponding number of straight segments. The approximation sufficiently handles multidimensional multiextreme functions by interpolating their discrete values accurately. Subsequently, the maximal magnetic field value is evaluated by minimizing the negative multiquadric approximation via a stochastic optimization method - differential evolution. Therefore, the procedure provides the maximal field value assessment on the basis of the limited number of computed discrete values, thus reducing the computational cost.
\end{abstract}

\section{Introduction}

An assessment of extremely low frequency (ELF) electromagnetic fields to which human beings are exposed has been of great interest for many years. Reason for this is the public concern regarding the possible health risk due to human exposure to such field sources. Moreover, there is a possibility of electromagnetic interference (EMI) threat concerning possible malfunction of sensitive electronic equipment. Namely, malfunction of some medical devices could seriously affect human health. Consequently, an assessment of the maximal ELF field quantities for comparison purposes with the (inter)national guidelines should be considered as a rather important task. Therefore, a particular attention should be focused to significant ELF field sources such as power substations. 
This paper deals with the magnetic fields generated by power substations. It is to be noticed that the research has been focused only toward the technical aspects of the field assessment, not considering health effects. ELF magnetic fields from power substations have been assessed via calculations or measurement procedures in many papers. Some of them are highlighted in this work [1-5], presenting a number of three-dimensional as well as contour plots. Nevertheless, they provided a useful description of the field characteristics, no technique incorporating certain optimization method for assessing the maximal magnetic field value has been applied in these papers. Spatial magnetic field function may have a number of local extremes arising from the fact that substation represents a concentration of magnetic field sources.

Consequently, the application of a stochastic optimization method differential evolution [6-8], which is in the scope of this work, could be a useful tool for the maximal field value assessment. The stochastic optimization method shows the well-known advantages over the gradient-based methods, namely: (i) the parallel global minimum search technique, (ii) the simplified set-up of optimization task and (iii) the ability to find global minimum. The optimization procedure is carried out using the multiquadric approximation $[9,10]$ of magnetic field, since the computed values are distributed in a set of discrete points through the volume of interest. The approximation based on radial basis functions is capable to handle multidimensional multiminima functions by interpolating their discrete data accurately.

\section{Magnetic field computation in a set of discrete points}

If linear mediums are concerned, the ELF magnetic field value in each spatial point can be assessed superposing the contributions of all conductors divided in a certain number of straight segments [1-5]. A $k$-th straight segment with the current $i_{k}$ placed in the Cartesian three-dimensional co-ordinate system is shown in Figure 1. Using the Biot-Savart law the magnetic field value in the point $C$, generated by the current $i_{k}$, can be written by:

$$
B_{k}(t)=\frac{\mu i_{k}(t)}{4 \pi C D}\left(\frac{A D}{A C}+\frac{D B}{B C}\right)
$$

where the included distances are presented in Figure 1, while $\mu$ is the magnetic permeability.

The corresponding magnetic field components produced by the current of the $k$-th straight segment are given by:

$$
\begin{aligned}
& B_{x, k}(t)=\cos \alpha_{B} \cdot B_{k}(t), \\
& B_{y, k}(t)=\cos \beta_{B} \cdot B_{k}(t), \\
& B_{z, k}(t)=\cos \gamma_{B} \cdot B_{k}(t),
\end{aligned}
$$




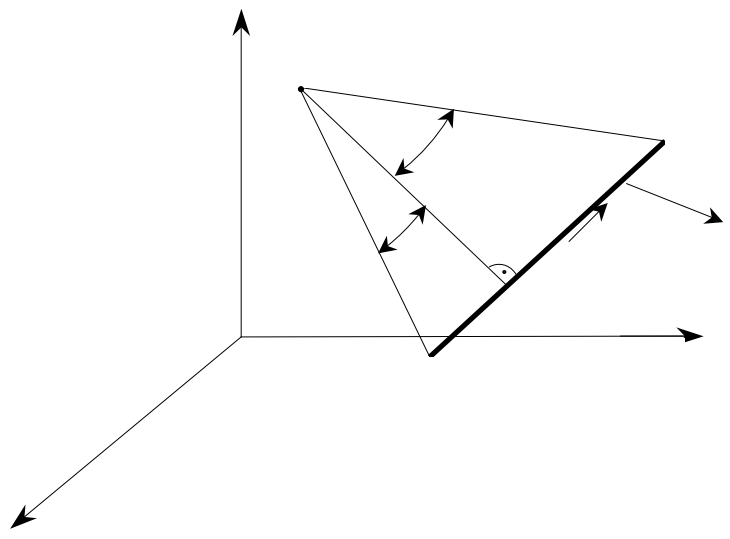

Figure 1: Straight segment in the Cartesian system.

where $\alpha_{B}, \beta_{B}$ and $\gamma_{B}$ are the angles defined by the magnetic field vector $\vec{B}_{k}$ and co-ordinate axis $x, y$ and $z$, respectively.

The total field components produced by the currents of $n$ segments are assembled by adding the contributions of each segment. Hence, the total field value in a chosen spatial point written in the Cartesian three-dimensional coordinate system is:

$$
B(t)=\sqrt{\left(\sum_{i=1}^{n} B_{x, i}(t)\right)^{2}+\left(\sum_{i=1}^{n} B_{y, i}(t)\right)^{2}+\left(\sum_{i=1}^{n} B_{z, i}(t)\right)^{2}} .
$$

\section{Maximal field value assessment}

The procedure for assessing the maximal magnetic field value generated by a power substation is outlined in a few steps: (A) multiquadric approximation of magnetic field, (B) minimization of objective function.

\subsection{Multiquadric approximation}

The vector of independent parameters, represented by the space co-ordinates, can be written by:

$$
\mathbf{P}=\left[\begin{array}{lll}
x & y & z
\end{array}\right]^{t} .
$$

The parameters are generally subjected to both inequality and equality constraints:

$$
\begin{gathered}
g_{m}(\mathbf{P}) \geq 0, m=1,2, \ldots, n_{t 1}, \\
h_{m}(\mathbf{P})=0, m=n_{t 1}+1, n_{t 1}+2, \ldots, n_{t 1}+n_{t 2},
\end{gathered}
$$


where $n_{t 1}, n_{t 2}$ are the total numbers of inequality and equality constraints, respectively.

Applying the procedure, a vector related with the maximal magnetic field value is assessed, fulfilling the imposed constraints. The computation of discrete field values would require rather high effort, if applied to a large number of parameter vectors. Hence, the procedure of the maximum assessment is carried out using the multiquadric approximation $[9,10]$ of magnetic field.

The approximation is determined by an expression:

$$
B_{M Q}(\mathbf{P})=\sum_{j=1}^{M_{p}} c_{j} r(\mathbf{P})_{j},
$$

where: $M_{p}$ is the total number of sample points $\mathbf{P}_{\mathrm{j}}$, having the computed values $B_{j}\left(\mathbf{P}_{\mathrm{j}}\right)$ and

$c_{j}$ stands for the approximation coefficients, while

$$
r(\mathbf{P})_{j}=\sqrt{\left\|\mathbf{P}-\mathbf{P}_{j}\right\|^{2}+h_{z}}, j=1,2, \ldots, M_{p}
$$

denotes radial basis functions, where $\left\|\mathbf{P}-\mathbf{P}_{j}\right\|$ is the Euclidean norm and $h_{z}$ is the shift parameter.

The value of $M_{p}$ should be a compromise between opposite requirements: the higher accuracy of the multiquadric approximation and the lower computational effort. The shift parameter $h_{z}$ can be determined by means of a few additional samples used for the error estimation.

The unknown coefficients $c_{j}$ are calculated from the matrix equation:

$$
\mathbf{c}=\mathbf{r}^{-1} \cdot \mathbf{B}
$$

where:

$$
\begin{gathered}
r_{i j}=\sqrt{\left\|\mathbf{P}_{i}-\mathbf{P}_{j}\right\|^{2}+h_{z}}, i, j=1,2, \ldots, M_{p}, \\
\mathbf{B}=\left[\begin{array}{llll}
B_{1}\left(\mathbf{P}_{1}\right) & B_{2}\left(\mathbf{P}_{2}\right) & \cdots & B_{M_{p}}\left(\mathbf{P}_{M_{p}}\right)
\end{array}\right]^{\mathrm{t}} .
\end{gathered}
$$

Hence, the discrete magnetic field data, obtained by the computation, are related with $M_{p}$ sample points only, thus reducing the required computational effort of the optimization procedure.

\subsection{Minimization of objective function}

Minimization of the objective function $-B_{M Q}(\mathbf{P})$, resulting in the maximal magnetic field value assessed, is formulated by: 


$$
\min \left(-B_{M Q}(\mathbf{P})\right)
$$

In order to perform the minimization procedure, i. e. to find the global minimum, a stochastic optimization method - differential evolution (DE) is applied [6-8].

A constant number $n_{p}$ of parameter vectors, representing members of a population, is used in each generation $G$ :

$$
\mathbf{P}_{i, G}, i=0,1, \ldots, n_{p}-1 .
$$

The population of the first generation is chosen randomly via rand function. There are several variants of $\mathrm{DE}$ algorithms. The one, using the best population vector to generate a new population member, is selected as a part of the proposed procedure. Hence, for each $\mathbf{P}_{i, G}$ there is the corresponding vector:

$$
\mathbf{V}_{i, G+1}=\mathbf{P}_{b e s t, G}+F\left(\mathbf{P}_{r_{1}, G}-\mathbf{P}_{r_{2}, G}\right),
$$

where: $\mathbf{P}_{b e s t, G}$ is the member having the lowest objective function value of the generation $G$,

$r_{1}, r_{2}$ denote randomly adopted integers from $\left[0, n_{p}-1\right]$,

$F$ is a real factor controlling the amplification of a weighted difference.

To enhance a new population diversity, the crossover of the vectors $\mathbf{V}_{i, G+1}$ and $\mathbf{P}_{i, G}$ is introduced, by which the new parameter vector:

$$
\mathbf{U}_{i, G+1}=\left[\begin{array}{llll}
u_{0 i, G+1} & u_{1 i, G+1} & \ldots & u_{(n-1) i, G+1}
\end{array}\right]^{t}
$$

is obtained as:

$$
\mathbf{U}_{i, G+1}=\left\{\begin{array}{l}
v_{j i, G+1} \text { for } j=\left\langle k_{n}\right\rangle_{n},\left\langle k_{n}+1\right\rangle_{n}, \ldots,\left\langle k_{n}+L_{n}-1\right\rangle_{n}, \\
p_{j i, G} \text { for all other } j \in[0, n-1]
\end{array}\right.
$$

where: $n$ is the total number of independent parameters,

\langle\rangle$_{n}$ stands for the modulo function with modulus $n$,

$k_{n}$ denotes the randomly chosen integer from $[0, n-1]$,

$L_{n}$ is the number of exchanged parameters from $[1, n]$.

The evaluation algorithm of $L_{n}$ is given by the following pseudo-code lines:

$$
\begin{aligned}
& L_{n}=0 ; \\
& \text { do }\{ \\
& \quad L_{n}=L_{n}+1 \\
& \text { \} while } \left.\left(\operatorname{rand}()<\operatorname{prob}_{C}\right) \text { and }\left(L_{n}<n\right)\right) ;
\end{aligned}
$$

where $\operatorname{prob}_{C}$ denotes the crossover probability used as a control variable.

If the resulting vector $\mathbf{U}_{i, G+1}$ gives the objective function value lower than the one corresponding to the vector $\mathbf{P}_{i, G}$, and if $\mathbf{U}_{i, G+I}$ fulfills the constraints, as well, it replaces $\mathbf{P}_{i, G}$ being a population member of the generation $G+1: \mathbf{P}_{i, G+1}=\mathbf{U}_{i, G+1}$. Otherwise, $\mathbf{P}_{i, G}$ is retained as a member of the generation $G+1$. 
The vector, related to the maximal value, equals the best vector assessed upon the prescribed number of generations:

$$
\mathbf{P}_{\max }=\mathbf{P}_{\text {best }, G_{l}},
$$

where $G_{l}$ is the number of the last generation.

The corresponding maximal magnetic field value from a power substation is then given by:

$$
B_{M Q}^{\max }\left(\mathbf{P}_{\max }\right)=\sum_{j=1}^{M_{p}} c_{j} r\left(\mathbf{P}_{\max }\right)_{j} .
$$

\section{Computational results}

The procedure presented so far is illustrated by an example of $110 / 10 \mathrm{kV} / \mathrm{kV}$ transmission substation of GIS (Gas-Insulated Substation) type in Split, Croatia. All the magnetic field levels $(f=50 \mathrm{~Hz})$ are computed at height $z=1 \mathrm{~m}$ above the ground. The high/middle voltage conductors are subdivided to several hundreds of straight segments, while their currents are computed on the basis of the total load of 97 MVA. The shieldings are disregarded, thus providing the results to take the "safe side" (the computed values are greater than the real ones).

There is no real possibility of public exposure within the transmission substation, while professional exposure is strictly limited to duration. Consequently, the computations are performed outside the fence of the substation, only. A simplified two-dimensional layout of the substation is shown in Figure 2.

Five domains where the greater magnetic field values are expected, can be seen in Figure 2. They are located in the vicinity of i) the underground cables, ii) the overhead lines and iii) the substation building. The associated multiquadric approximations of the magnetic field distributions are shown in Figures $3 \mathrm{a}-3 \mathrm{~d}$ leading to the conclusions carried out below.

Domain No.1:

The magnetic field values decay rapidly by going away from the cable route consisted of 6 three-core $10 \mathrm{kV}$ cables, Figure $3 \mathrm{a}$. The maximal field value of the domain is $B_{\max }=2.701 \mu T$.

Domain No.2:

The field intensity increases by approaching the overhead line route having the maximal value of $B_{\max 2}=0.945 \mu T$, Figure $3 \mathrm{~b}$.

Domain No.3:

The field values calculated right above $10 \mathrm{kV}$ underground cables ( 9 cable lines) are significantly greater than the other values, Figure $3 \mathrm{c}$. Also, there is a local intensity increase above the area where three cables change their direction in accordance with Figure 2. The maximal field value is $B_{\max 3}=3.344 \mu T$. 


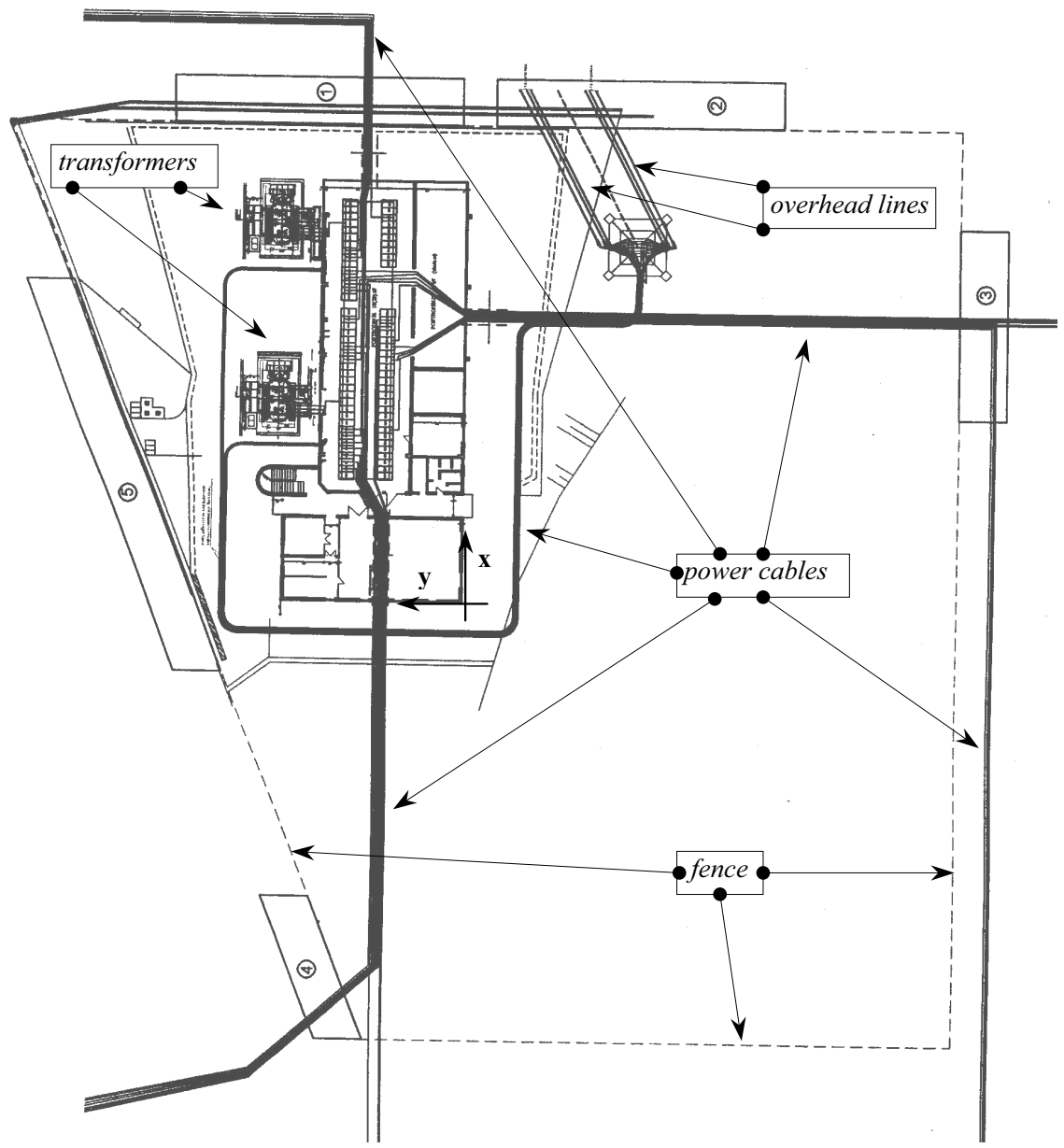

Figure 2: $\quad$ Substation layout.

Domain No. 4:

Similar distribution can be seen in Figure $3 \mathrm{~d}$ for the area No. 4. Greater field values are associated with the cable route consisted of 7 cable lines. The maximal value is $B_{\max 4}=2.552 \mu T$. A local field increase is visible in the corner for approaching the route of three lines going on in the other direction, Figure 2. Domain No. 5:

The field distribution over the area No. 5 is not presented, since no field value exceeds $0.75 \mu T$. The low field values are obtained as the underground cables are not buried there. 


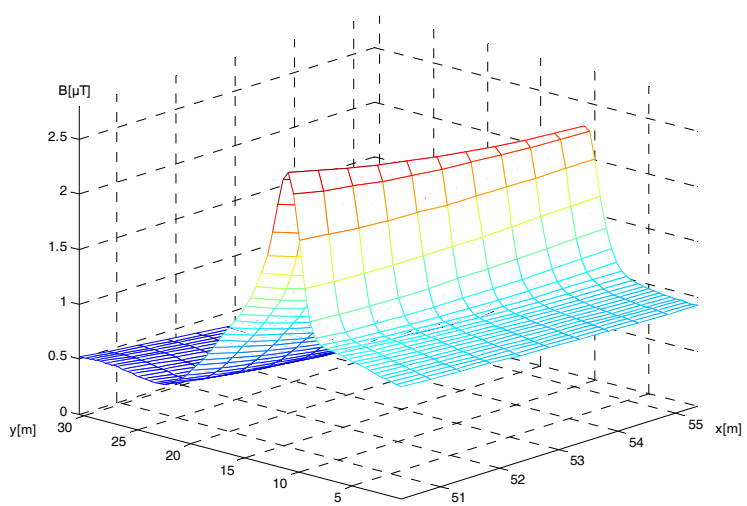

(a)

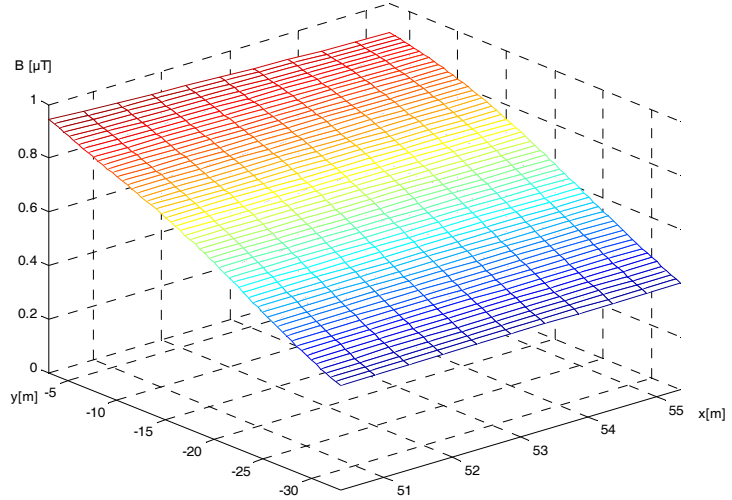

(b)

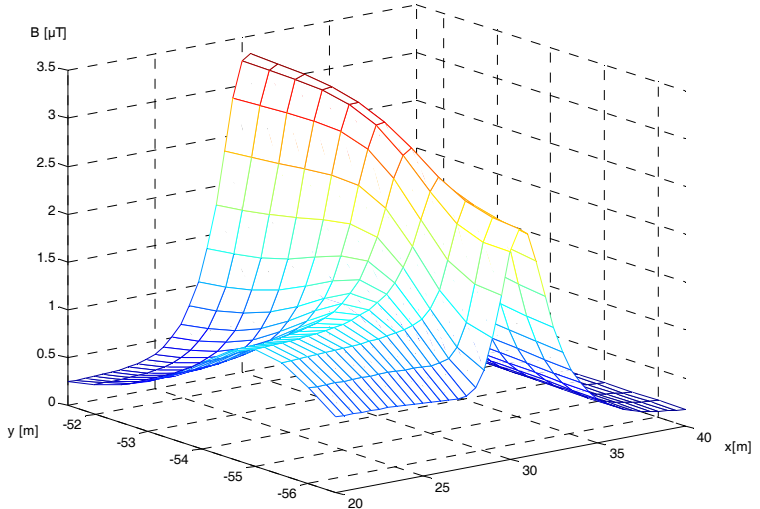

(c)

Figure 3: (a): The multiquadric approximation of the magnetic field - domain No. 1. (b): The multiquadric approximation of the magnetic field domain No. 2. (c): The multiquadric approximation of the magnetic field - domain No. 3. (d): The multiquadric approximation of the magnetic field - domain No. 4. 


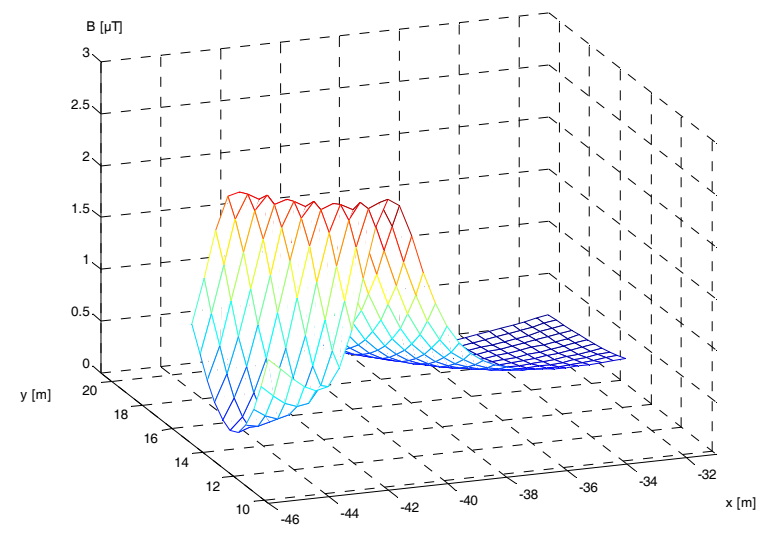

(d)

Figure 3: Continued.

\section{Concluding remarks}

A technique for the assessment of the maximal ELF magnetic field value from a power substation is presented in this work. The procedure is based on the multiquadric approximation of the magnetic field obtained using discrete field values. The values are calculated via the Biot-Savart law, where each energised substation conductor has been divided into a corresponding number of straight segments. The approximation is capable to handle multidimensional multiextreme functions by accurate interpolating their discrete values. Subsequently, the maximal value is assessed by minimizing the negative multiquadric approximation using the stochastic optimization method differential evolution. The presented procedure provides the maximal field value assessment on the basis of a limited number of the calculated values, thus reducing the computational cost.

\section{References}

[1] P. S. Wong, T. M. Rind, S. M. Harvey, R. R. Scheer: "Power Frequency Electric and Magnetic Fields from $230 \mathrm{kV}$ Gas-insulated Substation", IEEE Trans. on Power Delivery, 9(3), pp. 1494-1501, July 1994.

[2] N. Hayashi, K. Isaka, Y. Yokoi: Analysis of 60-Hz Magnetic Fields near Ground Level in 187-kV Switchyard of a 187/66-kV AC Substation, IEEE Trans. on Power Delivery, Vol. 7, No. 1, pp. 237-244, January 1992.

[3] N. Hayashi, K. Isaka, Y. Yokoi: ELF Electromagnetic Environment in Power Substations, Bioelectromagnetics, Vol. 10, pp. 51-64, 1989.

[4] Geri, M. Veca: Power-frequency Magnetic Field Calculation around an Indoor Transformer Substation, Boundary Elements XXVII, Incorporating Electrical Engineering and Electromagnetics, pp. 695-704, WIT Press, Southampton, Boston, 2005. 
[5] T. Keikko, S. Kuusiluoma, M. Suojanen, P. Menonen, L. Korpinen: Examples of Magnetic Field Calculations in Indoor Distribution Substations, Transactions on Modelling and Simulation, Vol. 30, pp. 927933, 2001.

[6] R. Storn, K. Price, 'Differential Evolution - A Simple and Efficient Adaptive Scheme for Global Optimization over Continuous Spaces, Technical Report TR-95-012, ICSI, Berkeley, USA, 1995.

[7] R. Storn, 'On the Usage of Differential Evolution for Function Optimization', NAFIPS, Berkeley, pp. 519-523, 1996.

[8] R. Storn, K. Price, 'Minimising the Real Functions of the ICEC'96 Contest by Differential Evolution', IEEE Conference on Evolutionary Computation, Nagoya, pp. 842-844, 1996.

[9] P. Alotto, A. Caiti, G. Molinari, M. Repetto, 'A Multiquadrics-based Algorithm for the Acceleration of Simulated Annealing Optimization Procedures, IEEE Trans. on Magnetics, 32(3), pp. 1198-1201, 1996.

[10] P. Alotto, M. Gaggero, G. Molinari, M. Nervi, 'A Design of Experiment and Statistical Approach to Enhance the Generalised Response Surface Method in the Optimization of Multiminima Problems, IEEE Trans. on Magnetics, 33(2), pp. 1896-1899, 1997. 